\title{
Schaltbare Oberflächen für Biosensoren
}

Für die weitere Erforschung intelligenter Oberflächen erhält die Kieler Naturwissenschaftlerin Prof. Martina Gerken eine

finanzielle Unterstützung von 1,5 Mio. Euro. Im Rahmen des

Projekts PhotoSmart sollen in den kommenden fünf Jahren mittels

Lichtreizen ein- und ausschaltbare Oberflächen entwickelt werden.

M it Hilfe des Zuschusses aus dem Fördertopf des Europäischen Forschungsrates (ERC) kann die Professorin für Nanotechnologie, optische Technologien und integrierte Systeme ihre Forschungsarbeiten weiterführen. Ziel ist am Ende eine Firmen-Neugründung.

Im Mittelpunkt der Arbeiten stehen intelligente Oberflächen, die in bewegliche, das heißt tragbare Sensoren eingebaut werden sollen. Diese Oberflächen sollen bestimmte Eigenschaften besitzen, beispielsweise von hydrophil zu hydrophob wechseln können. Gerken kennt bereits einige passende $\mathrm{Ma}$ terialien mit denen diese Eigenschaften erzielt werden können. Weiter werden bestimmte Materialien mit einer bindenden beziehungsweise nicht-bindenden Oberfläche für unterschiedliche Anwendungsbereiche gesucht.

Da es sich bei diesen Materialien um Biomaterialien handelt, ist es naheliegend, dass eine enge Verbindung zum Forschungsbereich „Nanowissenschaften und Oberflächenforschung “ an der Christian-Albrechts-Universität hergestellt wird.

\section{Binden oder loslösen mittels Schaltermoleküle}

Ausgangspunkt ist die Erkenntnis, dass Oberflächen mittels winziger SchalterMoleküle binden oder loslösen können. Teil der Forschungsarbeiten wird es auch sein, Gele zu finden, die sich für einen Einsatz auf risikobehafteten Oberflächen eignen. Die Lichtquel- len, die für die Umschaltung von hydrophil auf hydrophob verantwortlich sind, müssen direkt auf die Oberflächen aufgebracht werden. Auch sollen sie den Transport durch die Flüssigkeit beleuchten, das heißt transparent machen können. Als Lichtquellen werden Laser oder Leuchtdioden eingesetzt, die wie in Displays funktionieren.

Als herausragender Forschungsteil von PhotoSmart sollen schließlich Wege gefunden werden, mit deren Hilfe es gelingt, beide Arten solcher intelligenter Oberflächen - hydrophil und hydrophob - sowie ihre schaltbaren Eigenschaften in einen Sensor zu integrieren. Diese Lichquelle muss ein sicheres Schalten bei geringer Leistungsaufnahme gewährleisten. Vorzugsweise sollen deshalb ultraviolette und blaue organische Leuchtdioden, kombiniert mit Nanostrukturen, auf den betreffenden Oberflächen eingesetzt werden.

Erste Vorstellungen von der praktischen Anwendung solcher Biosensoren sind bereits vorhanden. So könnten Mikrofluidikchips hergestellt werden, die dank der Schaltmöglichkeit hydrophil/hydrophob programmierbar sind. Solche Chips könnten genutzt werden, um die Bewegung von Wasser- oder Flüssigkeitstropfen in miniaturisierten Sensoren zu steuern oder zu kontrollieren. Würde man die Eigenschaften bindend/nicht bindend hervorheben, wäre die Herstellung innovativer, hochsensibler und multifunktioneller Biosensoren möglich.

Richard E. Schneider

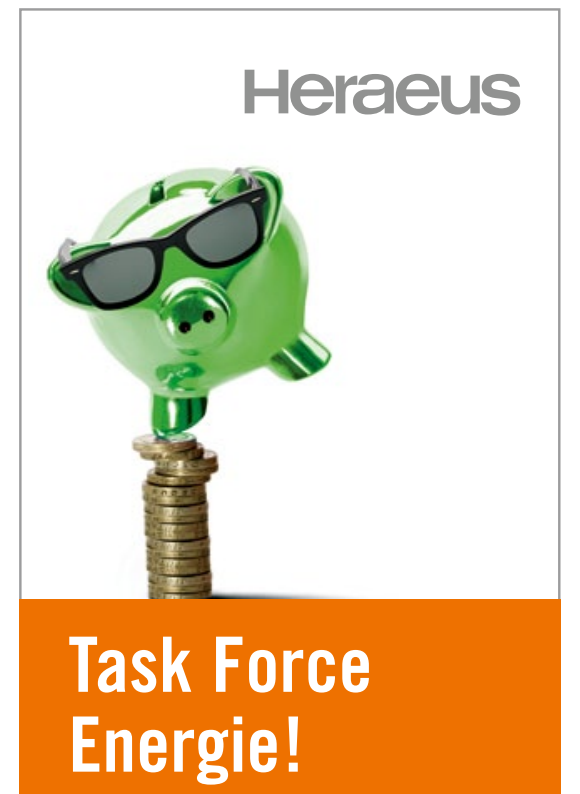

Kann der Lack schneller trocknen?

Kann der Ausschuss reduziert werden? Ist der Energieverbrauch zu hoch? Manchmal lohnt es sich, die gesamte Anlage auf den Prüfstand zu stellen! Denn Optimierung ist oft wesentlich sinnvoller und kostengïnstiger als eine Neuanschaffung. Unser Consulting Team hilft, Platz, Zeit und Energie zu sparen.

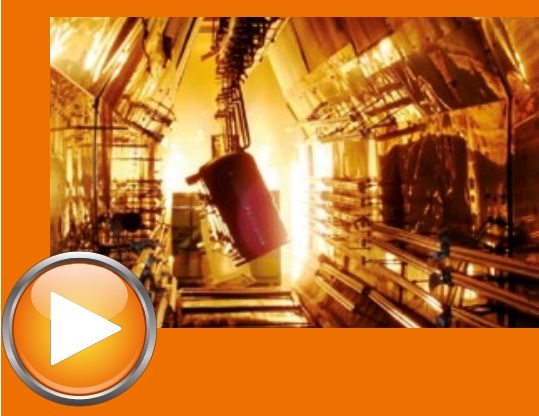

Ihr Kontakt zu unserer Task Force Energie:

hng-infrared@heraeus.com

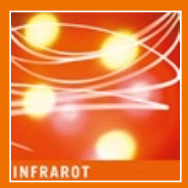

\section{Gut für Ihren}

Wettbewerbsvorteil.

Heraeus Noblelight GmbH

www.heraeus-noblelight.com/infrared

HANNOVER MESSE Halle 3 Stand E14 